Artículo científico

Volumen 31(3):581-595. Septiembre-diciembre, 2020 e-ISSN 2215-3608, doi:10.15517/am.v31i3.38999 http://www.revistas.ucr.ac.cr/index.php/agromeso

\title{
Altoandina: nueva variedad de avena forrajera para la zona Andina en Colombia ${ }^{1}$
}

\section{Altoandina: new variety of forage oats for the Andean area in Colombia}

\author{
Luis F. Campuzano-Duque ${ }^{2}$,Edwin Castro-Rincón ${ }^{3}$, Javier Castillo-Sierra ${ }^{4}$, Daniel Torres-Cuesta ${ }^{4}$, \\ David Nieto-Sierra ${ }^{3}$, Paola A. Portillo-Lopez ${ }^{3}$
}

1 Recepción: 17 de setiembre, 2019. Aceptación: 20 de mayo, 2020. Este trabajo formó parte de un Proyecto asociado al Programa de Mejoramiento de Forrajes en Colombia y financiado por el Ministerio de Agricultura y Desarrollo Rural (MADR) y la Corporación Colombiana de Investigación Agropecuaria (AGROSAVIA). Colombia.

2 Corporación Colombiana de Investigación Agropecuaria (AGROSAVIA). Centro de Investigación La Libertad, Villavicencio, Colombia. lcampuzano@agrosavia.co (autor para correspondencia; https://orcid.org/0000-0003-1146-4591).

3 Corporación Colombiana de Investigación Agropecuaria (AGROSAVIA). Centro de Investigación Obonuco, Pasto, Colombia. ecastro@ agrosavia.co (https://orcid.org/0000-0001-9841-8242); dnieto@agrosavia.co (https://orcid.org/0000-0003-4808-1201); pportillo@ agrosavia.co (https://orcid.orgg/0000-0003-1189-9173).

4 Corporación Colombiana de Investigación Agropecuaria (AGROSAVIA). Centro de Investigación Tibaitatá, Bogotá, Colombia. jcastillo@ agrosavia.co (https://orcid.org/0000-0003-0797-3908); dtorres@agrosavia.co (https://orcid.org/0000-0001-9101-0543).

\section{Resumen}

Introducción. La evaluación y selección de nuevas especies forrajeras con atributos apropiados de producción y calidad es una estrategia segura y eficaz para mejorar la eficiencia de los sistemas de producción de leche en Colombia. Objetivo. Describir el proceso de desarrollo y las principales características de Altoandina, nueva variedad de avena forrajera. Materiales y métodos. Durante el segundo semestre de 2016 se realizó una prueba de evaluación agronómica (PEA) en cuatro localidades de la subregión Altiplano Cundiboyacense y en cuatro localidades de la subregión Altiplano de Nariño, en un diseño de bloques completos al azar, con dos genotipos de avena forrajera AV25-S y AV25-T y tres variedades (Avenar, Cajicá, Cayuse). Resultados. AV25-S y AV25-T presentaron: tolerancia al volcamiento, a diferencia de los tres testigos que mostraron un volcamiento superior al $30 \%$; resistencia a la roya de la hoja y del tallo y rendimiento de forraje verde de 64,6 y 65,3 $\mathrm{t} \mathrm{ha}^{-1}$, respectivamente, superior al obtenido en Avenar $(55,5)$, en Cajicá $(43,7)$ y en Cayuse $(59,1)$. Conclusión. AV25-T por su tolerancia a volcamiento, resistencia a la roya de la hoja y del tallo y rendimiento de forraje verde superior a las variedades comerciales, fue inscrita en el sistema nacional de cultivares como nueva variedad forrajera denominada Altoandina, con recomendación para la subregión Altiplano Cundiboyacense y Altiplano de Nariño.

Palabras clave: adaptación, Avena sativa, interacción genotipo ambiente, producción de forraje.
Abstract
Introduction. The evaluation and selection of new fodder species with appropriate production and quality attributes is a safe and effective strategy to improve the efficiency of milk production systems in Colombia. Objective. To describe the development process and the main characteristics of Altoandina, a new variety of fodder oats. 
Materials and methods. During the second half of 2016, an agronomic assessment test (PEA) was carried out in four locations in the Altiplano Cundiboyacense subregion and in four locations in the Altiplano of Nariño subregion, in a randomized complete block design, with two genotypes of fodder oats AV25-S and AV25-T and three varieties (Avenar, Cajica, Cayuse). Results. AV25-S and AV25-T showed: tolerance to overturn unlike the three controls that showed a overturn of more than 30\%, resistance to leaf and stem rust, and green forage performance of 64.6 and 65.3 $\mathrm{t} \mathrm{ha}^{-1}$, respectively, higher than that obtained in Avenar (55.5), Cajica (43.7) and Cayuse (59.1). Conclusion. AV25-T for its tolerance to overturning, resistance to leaf and stem rust and green fodder yield superior to commercial varieties, was registered in the national cultivar system as a new fodder variety called Altoandina, with recommendation for the Cundiboyacense Altiplano and Nariño Altiplano.

Keywords: adaptation, Avena sativa, genotype environment interaction, forage production.

\section{Introducción}

La ganadería de leche especializada en Colombia muestra en los departamentos de Cundinamarca, Boyacá y Nariño un total de 149461 fincas, con la particularidad que el $96 \%$ de estas están en condiciones de minifundio, con extensiones entre 1 y 50 ha (144 592 fincas). Del total de bovinos de leche en el país (3 998 373), estos tres departamentos poseen el 23,7\% con 948141 bovinos hembras (DANE, 2015).

En el modelo de empresa ganadera especializada en leche en el Trópico Alto Colombiano, el componente de alimentación está sustentado en la oferta de pasturas mejoradas naturalizadas, principalmente con el pasto Kikuyo (Cenchrus clandestinus H.) (Cuesta, 2005; Sossa y Barahona, 2015; Arango et al., 2017; Vargas et al., 2018). No obstante, los problemas estacionales del clima, principalmente en la época de verano y en heladas, representan una perdida en la cantidad y calidad del forraje (Cuesta, 2005; Carulla y Ortega, 2016; Vargas et al., 2018). Para afrontar la reducción de la oferta forrajera, el uso de especies principalmente de cereales como el maíz y la avena (Avena sativa) es una buena opción. Como forraje, la avena tiene alta digestibilidad y energía metabolizable y su fibra presenta mejores cualidades que otros cereales de grano pequeño; y el grano, presenta alta cantidad y calidad de proteínas, carbohidratos, minerales (hierro), grasas y vitamina B (Ramírez-Ordoñez et al., 2013; Montaño-Carrasco et al., 2017; Mamani y Cotacallapa, 2018).

Las variedades comerciales de avena forrajera actuales utilizadas por los productores de leche en Colombia en la zona Andina, presentan susceptibilidad al volcamiento y a la roya de la hoja y del tallo, que comprometen el rendimiento de forraje ocasionando pérdidas hasta en un $60 \%$ y detrimento en la calidad (Wise y GoblemanWerner, 1993; Cuesta, 2005). Asimismo, la variedad comercial Cayuse, de mayor uso actual en el país es tardía con 160 días a floración y 180 días a cosecha de forraje verde (Cuesta, 2005). Puccinia coronata f. sp. avenae y Puccinia graminis $f$. sp. avenae Erikss and Hennig, como causantes de la roya de la hoja y del tallo en avena, respectivamente, son consideradas a nivel mundial como los patógenos más destructivos, afectando el rendimiento y la calidad del forraje en las principales áreas productoras (Berlín et al., 2013; Keiper et al., 2006).

La Corporación Colombiana de Investigación Agropecuaria (AGROSAVIA), desarrolla investigación en forrajes para el Trópico Alto Colombiano, con el fin de identificar nuevos genotipos forrajeros con atributos agronómicos y productivos deseables como la tolerancia al volcamiento y con menor incidencia de roya de la hoja y del tallo. Por lo cual, se realizó una prueba de evaluación agronómica (PEA) en la subregión Altiplano Cundiboyacense y Altiplano de Nariño, para determinar el grado de adaptación y el comportamiento agronómico de dos genotipos promisorios de avena (AV025-S y AV025-T) en comparación con tres variedades comerciales de avena forrajera (Cayuse, Avenar y Cajicá).

El objetivo fue describir el proceso de desarrollo y las principales características de Altoandina, nueva variedad de avena forrajera. 


\section{Materiales y métodos}

\section{Localización}

Durante el segundo semestre de 2016, se evaluaron, en condiciones de campo, dos genotipos de avena forrajera (Avena sativa), en la región Andina, en cuatro localidades de la subregión Altiplano Cundiboyacense y cuatro localidades de la subregión Altiplano de Nariño, mediante una prueba de evaluación agronómica (PEA) ajustada a la Resolución 01985 (ICA, 2000).

Las localidades y su ubicación (municipio y georreferenciación) se identificaron como: Localidad 1 (MO):

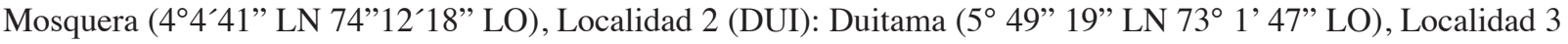

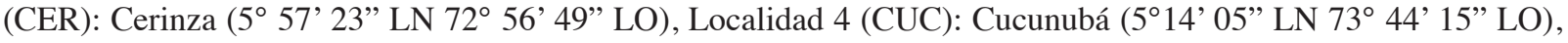

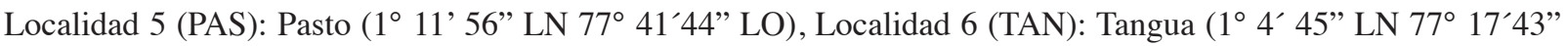

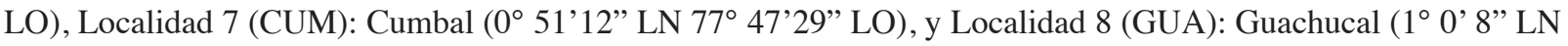
$\left.77^{\circ} 46^{\prime} 53^{\prime \prime} \mathrm{LO}\right)$.

Las localidades se ubicaron en altitudes entre 2543 a $2760 \mathrm{~m}$, con una temperatura media anual entre 16,9 a $17,4^{\circ} \mathrm{C}$, en la subregión altiplano Cundiboyacense y de 2710 a $3184 \mathrm{~m}$ con una temperatura media anual entre 12,6 a $14,5^{\circ} \mathrm{C}$ en el altiplano de Nariño. Con base en estas dos variables de clima y según la clasificación Caldad-Lang (Gutiérrez, 1991), todas las localidades se clasificaron como Fsh (clima frío semihúmedo) con excepción de CUM que se clasificó como Mfh (clima muy frío húmedo). La principal diferencia entre localidades, en relación con el clima, desde germinación hasta la cosecha de forraje verde, fue la precipitación. De acuerdo con el requerimiento de $400 \mathrm{~mm}$ para la producción de 60 a $80 \mathrm{t} \mathrm{ha}^{-1}$ de forraje verde en avena (Bhatti et al., 1992; Hussain et al., 2010; Terán-Chaves et al., 2014), seis de las ocho localidades MOS, CUC, CER, PAS, CUM y GUA se satisfizo el agua requerida en el $100 \%$ o muy cercana al $100 \%$ y en dos localidades DUI y TAN presentaron déficit de agua del 25 $\%$ y $43 \%$, respectivamente, siendo más severa en la última localidad (Cuadro 1).

Cuadro 1. Altitud, precipitación y temperatura media ocurrida entre la emergencia a cosecha de forraje verde de avena (Avena sativa), en ocho localidades de la zona Andina colombiana. Corporación Colombiana de Investigación Agropecuaria (AGROSAVIA). $2016-2017$.

Table 1. Altitude, precipitation, and average annual temperature between the emergence of oats (Avena sativa) green forage harvest, in eight localities in the Colombian Andean zone. Corporación Colombiana de Investigación Agropecuaria (AGROSAVIA). $2016-2017$.

\begin{tabular}{lccccc}
\hline Subregión & Localidad & Altitud Msnm & $\begin{array}{c}\text { Precipitación } \\
\mathbf{m m}\end{array}$ & $\begin{array}{c}\text { Agua } \\
\text { satisfecha* \% }\end{array}$ & Temperatura ${ }^{\circ} \mathbf{C}$ \\
\hline Altiplano Cundiboyacense & Mosquera & 2543 & 618,0 & 100 & 17,4 \\
& Cucunubá & 2550 & 386,8 & 97 & 16,9 \\
& Duitama & 2760 & 300,0 & 75 & 17,2 \\
& Cerinza & 2854 & 526,8 & 100 & 17,1 \\
\hline Altiplano de Nariño & Pasto & 2710 & 380,6 & 95 & 14,5 \\
& Tangua & 2999 & 210,5 & 53 & 15,2 \\
& Cumbal & 3184 & 379,7 & 95 & 12,6 \\
& Guachucal & 3180 & 398,7 & 99 & 12,4 \\
\hline
\end{tabular}

Fuente: Datos climatológicos IDEAM (2017).

*: porcentaje de agua satisfecha sobre la base de requerimiento de $400 \mathrm{~mm}$ (Batti et al., 1992; Hussain et al., 2010; Terán-Chaves et al., 2014) / Source: IDEAM Climatological data (2017). *: percentage of water satisfied based on 400 mm requirement (Batti et al., 1992; Hussain et al., 2010; Terán-Chaves et al., 2014). 


\section{Materiales genéticos y diseño experimental}

La prueba de evaluación agronómica (PEA) estuvo constituida por dos genotipos promisorios de avena forrajera del programa de mejoramiento genético de la Corporación Colombiana de Investigación Agropecuaria (AGROSAVIA), seleccionados por su rendimiento de forraje verde, tolerancia al volcamiento y resistencia a roya de la hoja y del tallo, en ensayos previos, en las condiciones de suelos de origen volcánico del orden de los Inceptisoles del Altiplano Cundiboyacense y Altiplano de Nariño e identificados experimentalmente como: AV25-S y AV25-T. Se utilizó un diseño de bloques completos al azar (DBCA) con cinco tratamientos (dos genotipos y tres variedades) y cuatro repeticiones. Las variedades utilizadas como testigo fueron: Avenar, Cajicá y Cayuse de uso comercial actual por los productores en Colombia, cuya principal debilidad está en la susceptibilidad a la roya de la hoja y del tallo y volcamiento. La unidad experimental estuvo constituida por parcelas de $30 \mathrm{~m}^{2}$ con una densidad de siembra de $80 \mathrm{~kg} \mathrm{ha}^{-1}$.

\section{Características del suelo y manejo agronómico}

Para conocer el nivel de fertilidad del suelo y la recomendación de correctivos y de fertilización, en cada localidad se tomaron muestras de suelo a una profundidad de 0 a $20 \mathrm{~cm}$, que permitió determinar las características físicas: textura (método del hidrómetro o Bouyoucos) y químicas: pH (método potenciómetro), materia orgánica (\%) (método de Walkley y Black), fósforo $\left(\mathrm{mg} \mathrm{kg}^{-1}\right)$ (método Bray II), calcio (Ca), magnesio (Mg) y potasio (K), expresados en $\left(\mathrm{cmol} \mathrm{kg}^{-1}\right)$ (extracción con acetato de amonio pH 7 y cuantificación por absorción atómica). Estos análisis se realizaron en el laboratorio de química de suelos, aguas y plantas de AGROSAVIA con la Norma Técnica de Calidad NTC-2005.

Para mejorar la condición de $\mathrm{pH}$ del suelo en cada localidad se aplicaron $4 \mathrm{t} \mathrm{ha}^{-1}$ de cal dolomítica. La preparación del suelo en cada localidad se realizó con un pase de arado de cincel vibratorio, dos pases de rastra y un pase de rastra pulidor. La siembra se realizó de forma manual al voleo y en cada localidad se realizó la fertilización basada en los resultados del análisis del suelo y el requerimiento nutricional de avena forrajera para producir 80 $\mathrm{t} \mathrm{ha}^{-1}$. Se aplicaron compuestos simples en $\mathrm{kg}^{-1} \mathrm{ha}^{-1} \mathrm{~N}: 150, \mathrm{P}_{2} \mathrm{O}_{5}: 120 ; \mathrm{K}_{2} \mathrm{O}: 90$, Ca: 70, Mg: 25 y Borozinco: 20. El nitrógeno se aplicó fraccionado a la siembra y macollamiento (75:75). Se realizó control de malezas, principalmente de gramíneas y de hoja ancha, desde el momento de la siembra con una aplicación de preemergente (ácido-4-amino-3,5,6-tricloropicolinico $200 \mathrm{cc} \mathrm{ha}^{-1}$ ) y posemergente (Metsulfuron Metil $15 \mathrm{~g} \mathrm{ha}^{-1}$ ). No se realizó control de insectos-plaga y patógenos con el fin de determinar el comportamiento de los genotipos en condiciones de inóculo natural, especialmente para roya de la hoja (Puccinia coronata $f$. sp avenae) y roya del tallo (Puccinia graminis $f$. sp avenae Erikss y Henning).

Los suelos asociados a las localidades de evaluación de los genotipos de avena forrajera por ubicación y geomorfología se clasificaron: a) altiplano Cundiboyacense (MOS, DUI, CER y CUC) como suelos ácidos del orden Inceptisoles y b) altiplano de Nariño (PAS, TAN, CUM y GUA) como suelos ácidos del orden Inceptisoles suborden Andeps, por su formación de origen volcánico con alto contenido de alófana (Ramos, 2017). En el altiplano Cundiboyacense predominaron texturas franco arcillosa y franco arcilloso arenosa, ligeramente ácido $(5,7)$, con contenido medio de materia orgánica $(5,1$ a $7,7 \%)$ y un contenido alto de fósforo $\left(20,5\right.$ a $\left.38,9 \mathrm{mg} \mathrm{kg}^{-1}\right)$. Los suelos del Altiplano de Nariño con una textura franco-arcillosa, un pH muy ácido $(5,2$ a 5,9), con un contenido alto de fósforo $\left(17,15\right.$ a $\left.25,2 \mathrm{mg} \mathrm{kg}^{-1}\right)$ y alto de materia orgánica $(8,7$ a 16,6 \%). En relación con el calcio, magnesio

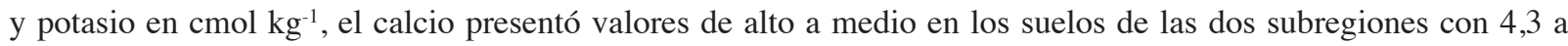
15,2 para el altiplano Cundiboyacense y de 3,3 a 8,8 para el altiplano de Nariño; magnesio con valores de bajo a alto en la subregión Cundiboyacense $(0,3$ a 3,6$)$ y de bajo a medio en la subregión Altiplano de Nariño $(0,6$ a 1,8$)$, y el potasio, con valores de bajo a alto en ambas subregiones de 0,1 a 0,9 en el Altiplano Cundiboyacense y de 0,2 a 0,7 en el Altiplano de Nariño (Cuadro 2). 
Cuadro 2. Características fisicoquímicas del suelo en ocho localidades de la zona Andina donde se evaluaron dos genotipos de avena forrajera (Avena sativa). Corporación Colombiana de Investigación Agropecuaria (AGROSAVIA). Colombia. 2016-2017.

Table 2. Physicochemical characteristics of the soil in eight localities of the Andean zone where two genotypes of forage oats (Avena sativa), were evaluated. Corporación Colombiana de Investigación Agropecuaria (AGROSAVIA). Colombia. 2016-2017.

\begin{tabular}{ccccccccc}
\hline Subregión & Localidad & Textura & $\mathbf{p H}$ & $\begin{array}{c}\text { Materia } \\
\text { orgánica } \%\end{array}$ & $\begin{array}{c}\text { Fósforo } \\
\mathbf{m g ~ k g}^{-1}\end{array}$ & $\begin{array}{c}\text { Calcio } \\
\mathbf{c m o l ~ k g}^{-1}\end{array}$ & $\begin{array}{c}\text { Magnesio } \\
\mathbf{c m o l ~ k g}^{-1}\end{array}$ & $\begin{array}{c}\text { Potasio } \\
\mathbf{c m o l}_{\mathbf{~ k g}} \mathbf{1}\end{array}$ \\
\hline \multirow{2}{*}{$\mathrm{ACB}$} & MOS & FAr & 5,7 & $5,1 \mathrm{M}$ & $24,7 \mathrm{~A}$ & $5,2 \mathrm{~A}$ & $3,6 \mathrm{~A}$ & $0,4 \mathrm{~A}$ \\
& DUI & FArA & 5,7 & $7,7 \mathrm{M}$ & $23,5 \mathrm{~A}$ & $4,9 \mathrm{~A}$ & $3,6 \mathrm{~A}$ & $0,9 \mathrm{~A}$ \\
& CER & FArA & 5,7 & $7,0 \mathrm{M}$ & $20,5 \mathrm{~A}$ & $4,3 \mathrm{~A}$ & $0,3 \mathrm{~B}$ & $0,1 \mathrm{~B}$ \\
\hline \multirow{2}{*}{$\mathrm{ADN}$} & CUC & FAr & 5,7 & $5,7 \mathrm{M}$ & $38,9 \mathrm{~A}$ & $6,4 \mathrm{~A}$ & $1,1 \mathrm{~B}$ & $0,6 \mathrm{~A}$ \\
& PAS & FAr & 5,9 & $8,9 \mathrm{~A}$ & $17,5 \mathrm{~A}$ & $8,8 \mathrm{~A}$ & $1,8 \mathrm{M}$ & $0,2 \mathrm{~B}$ \\
& TAN & FAr & 5,2 & $11,0 \mathrm{~A}$ & $17,1 \mathrm{~A}$ & $4,0 \mathrm{M}$ & $0,6 \mathrm{~B}$ & $0,7 \mathrm{~A}$ \\
& CUM & FAr & 5,2 & $16,6 \mathrm{~A}$ & $18,3 \mathrm{~A}$ & $7,4 \mathrm{~A}$ & $1,6 \mathrm{M}$ & $0,7 \mathrm{~A}$ \\
& GUA & FAr & 5,4 & $8,7 \mathrm{~A}$ & $25,2 \mathrm{~A}$ & $3,4 \mathrm{M}$ & $1,4 \mathrm{M}$ & $0,6 \mathrm{~A}$ \\
\hline
\end{tabular}

ACB: Altiplano Cundiboyacense; ADN: Altiplano de Nariño; MOS: Mosquera; DUI: Duitama; CER: Cerinza; CUC: Cucunubá; PAS: Pasto; TAN: Tangua; CUM: Cumbal; GUA: Guachucal; FAr: franco arcilloso; FArA: franco arcilloso arenoso; A: alto; M: medio; B: bajo (Riascos, 1998) / ACB: Altiplano Cundiboyacense; ADN: Altiplano de Nariño; MOS: Mosquera; DUI: Duitama; CER: Cerinza; CUC: Cucunubá; PAS: Pasto; TAN: Tangua; CUM=Cumbal; GUA: Guachucal; FAr: clay loam; FArA: sandy clay loam; A: high; M: medium; B: low (Riascos, 1998).

\section{Variables evaluadas}

Con base en la escala de las etapas de crecimiento de la avena, de códigos decimales (Z) (Zadocks et al., 1974), se determinaron las siguientes variables: a) días a floración (DF): determinada como el número de días a partir del momento de la emergencia hasta que el $50 \%$ de las plantas presentaron exposición de la panoja emergida y con anteras amarillas visibles (estado Z-6,5) y expresada en días, b) días a cosecha de forraje verde (DCFV): se determinó a partir del momento de la emergencia hasta la cosecha de forraje verde en estado grano lechoso y expresada en días, y c) volcamiento de tallo (VT): se determinó de forma visual, expresado en porcentaje y transformados (raíz cuadrada del arcoseno). Las variables DCFV y VT se determinaron en la etapa de grano lechoso $(\mathrm{Z}-7,0)$.

El rendimiento de forraje verde (RFV) se determinó en el estado de grano lechoso $(\mathrm{Z}-7,0)$ y expresada en $\mathrm{t}$ ha ${ }^{-1}$, con la cosecha de $15 \mathrm{~m}^{2}$ en cada unidad experimental. En relación con la roya de la hoja (Puccinia coronata $f . s p$ avenae) y la roya del tallo (Puccinia graminis $f$. $s p$ avenae Erikss y Henning) fueron evaluadas en la etapa grano en la zona central, extendidos $20 \%$ (Z-7,02) en una muestra de 50 plantas al azar. La roya de la hoja se terminó en el tercio superior de la planta y la roya del tallo, en los tallos. Se utilizó, para ambos patógenos, la escala propuesta por Peterson et al. (1948), donde se combina la severidad del área foliar afectada en porcentaje entre 0 y 100 y la reacción varietal (Cuadro 3).

\section{Análisis estadístico}

Mediante el procedimiento Proc Univariate de SAS, versión 9.3 (SAS Institute Inc, 2003). La comparación entre medias de los tratamientos se realizó mediante la prueba de Tukey $(\mathrm{p}=0,05)$. Con la variable rendimiento de forraje verde se realizaron los análisis de varianza individuales por localidad y combinando localidades. Para el análisis de varianza, las localidades y las repeticiones se consideraron efectos aleatorios y los genotipos, efectos fijos. 
Cuadro 3. Escala de reacción varietal a la roya de la hoja (Puccinia coronata f. sp avenae) y roya del tallo (Puccinia graminis f. sp avenae Erikss y Henning) en avena forrajera (Avena sativa).

Table 3. Varietal reaction scale to leaf rust (Puccinia coronata f. sp avenae) and stem rust (Puccinia graminis f. sp avenae Erikss and Henning) in forage oats (Avena sativa).

\begin{tabular}{lc}
\hline Reacción varietal * & Categoría \\
\hline Inmune: ausencia de uredos & $\mathrm{I}$ \\
Resistente: pocos y pequeños uredos & $\mathrm{R}$ \\
Moderadamente resistente: muchos uredos pequeños a medianos & $\mathrm{MR}$ \\
Moderadamente susceptible: numerosos uredos medianos en áreas cloróticas & $\mathrm{MS}$ \\
Susceptible: numerosos uredos, sin clorosis ni necrosis. & $\mathrm{S}$ \\
Altamente susceptible: numerosos uredos de gran tamaño sin clorosis ni necrosis & AS \\
\hline
\end{tabular}

* Peterson et al. (1948).

El modelo estadístico utilizado fue el siguiente: Yijk $=\mu+\mathrm{Gi}+\mathrm{Aj}+(\mathrm{GA}) \mathrm{ij}+\mathrm{Bk}(\mathrm{j})+$ Eijk; donde: Yijk $=$ rendimiento de forraje verde promedio del i-ésimo genotipo obtenido en el j-ésimo ambiente y k-ésima repetición; $\mu=$ efecto de la media general; $\mathrm{Gi}=$ efecto del i-ésimo genotipo; $\mathrm{Aj}$ = efecto del j-ésimo ambiente; $(\mathrm{GA}) \mathrm{ij}=$ efecto de la interacción entre el i-ésimo genotipo y el j-ésimo ambiente; $B k(j)=$ efecto de la k- ésima repetición en el j-ésimo ambiente y Eijk = efecto aleatorio del error experimental asociado al i-ésimo genotipo en el j-ésimo ambiente y K-ésima repetición, según el modelo lineal aditivo.

\section{Análisis de estabilidad fenotípica y criterio de selección del mejor genotipo}

La interacción genotipo x localidad fue estudiada para la variable RFV con base en el modelo de efecto principal aditivo e interacción multiplicativa (AMMI), descrito por Gabriel (1971) y Crossa et al. (2002). El criterio de selección del genotipo candidato a variedad fue el RFV, resistencia a la roya de la hoja y del tallo y tolerancia al volcamiento.

\section{Resultados}

\section{Días a floración (DF) y días a cosecha de forraje verde de avena (DCFV)}

Se presentaron diferencias altamente significativas $(\mathrm{p}<0,01)$ en los días a floración (DF) para genotipos en las localidades de MOS, CUC, DUI, TAN, CUM Y GUA y, diferencias significativas $(\mathrm{p}<0,05)$ para las localidades CER y PAS. Los genotipos AV25-T y AV25-S en cada localidad presentaron un promedio igual de $\mathrm{DF}(\mathrm{p}<0,01)$, pero diferentes $(\mathrm{p}<0,05)$ a los tres testigos comerciales en todas las localidades, con excepción de PAS, con valores de DF de 88 a 114 (Cuadro 4). Los testigos comerciales Avenar y Cajicá no presentaron diferencias estadísticas $(\mathrm{p}<0,01)$ entre ellos, con un valor de DF de 74 a 109 para Avenar y 68 a 108 para Cajicá, considerados como una floración intermedia. La variedad Cayuse presentó una floración tardía, diferente estadísticamente $(\mathrm{p}<0,01)$ a los demás genotipos en todas las localidades, con DF de 110 a 165 (Cuadro 4).

Los días a cosecha de forraje verde de avena (DCFV) presentaron diferencias altamente significativas $(\mathrm{p}<0,01)$ para los cinco genotipos en todas las localidades. Los genotipos AV25-S y AV25-T en cada localidad presentaron promedios iguales estadísticamente $(\mathrm{p}<0,01)$ entre sí, pero diferentes $(\mathrm{p}<0,05)$ a los tres testigos comerciales en 
Cuadro 4. Días a floración (DF) de dos genotipos promisorios y tres variedades comerciales de avena forrajera (Avena sativa), en ocho localidades de la zona Andina de Colombia. Corporación Colombiana de Investigación Agropecuaria (AGROSAVIA). 2016-2017.

Table 4. Days to flowering (DF) of two promising genotypes and three varieties of forage oats (Avena sativa), in eight localities in the Colombian Andean zone. Corporación Colombiana de Investigación Agropecuaria (AGROSAVIA). 2016-2017.

\begin{tabular}{|c|c|c|c|c|c|c|c|c|}
\hline \multirow[t]{3}{*}{ Genotipo } & \multicolumn{8}{|c|}{ Subregión/localidad } \\
\hline & \multicolumn{4}{|c|}{ Altiplano Cundiboyacense } & \multicolumn{4}{|c|}{ Altiplano de Nariño } \\
\hline & MOS & DUI & CER & CUC & PAS & TAN & CUM & GUA \\
\hline AV25-T & $94,5 \mathrm{~b}$ & $88,0 \mathrm{~b}$ & $95,0 \mathrm{~b}$ & $90,0 \mathrm{~b}$ & $109,0 \mathrm{~b}$ & $114,0 \mathrm{a}$ & $103,0 \mathrm{~b}$ & $104,0 \mathrm{~b}$ \\
\hline AV25-S & $94,5 \mathrm{~b}$ & $88,0 \mathrm{~b}$ & $95,0 \mathrm{~b}$ & $90,0 \mathrm{~b}$ & $109,0 \mathrm{~b}$ & $114,0 \mathrm{a}$ & $103,0 \mathrm{~b}$ & $104,0 \mathrm{~b}$ \\
\hline Avenar & $74,0 \mathrm{c}$ & $72,0 \mathrm{c}$ & $75,0 \mathrm{c}$ & $76,0 \mathrm{c}$ & $109,0 \mathrm{~b}$ & $97,3 \mathrm{~b}$ & $85,0 \mathrm{c}$ & $78,0 \mathrm{c}$ \\
\hline Cajicá & $68,0 \mathrm{c}$ & $68,0 \mathrm{c}$ & $70,0 \mathrm{c}$ & $72,0 \mathrm{c}$ & $108,0 \mathrm{~b}$ & $96,7 \mathrm{~b}$ & $85,0 \mathrm{c}$ & $78,0 \mathrm{c}$ \\
\hline Cayuse & $132,0 \mathrm{a}$ & $130,0 \mathrm{a}$ & $135,0 \mathrm{a}$ & $138,0 \mathrm{a}$ & $144,0 \mathrm{a}$ & $116,0 \mathrm{a}$ & $110,0 \mathrm{a}$ & $165,0 \mathrm{a}$ \\
\hline
\end{tabular}

a-b-c: Letras diferentes entre columnas indican diferencia estadística significativa $(\mathrm{p}<0,01)$. MOS: Mosquera; DUI: Duitama; CER: Cerinza; CUC: Cucunubá; PAS: Pasto; TAN: Tangua; CUM: Cumbal; GUA: Guachucal / a-b-c: different letters between columns indicate significant statistical difference ( $\mathrm{p}<0.01)$. MOS: Mosquera; DUI: Duitama; CER: Cerinza; CUC: Cucunubá; PAS: Pasture; TAN: Tangua; CUM: Cumbal; GUA: Guachucal.

todas las localidades, con valores de DCFV entre 111 a 165 días. Los testigos comerciales Avenar y Cajicá no presentaron diferencias estadísticas $(\mathrm{p}<0,01)$ en todas las localidades y mostraron valores de DCFV entre 88 a 121,8. La variedad Cayuse fue el testigo comercial que presentó una cosecha de forraje en verde más tardía, diferente estadísticamente $(\mathrm{p}<0,01)$ a los demás genotipos en todas las localidades con valores de DCFV entre 137,8 y 180,3 (Cuadro 5).

\section{Volcamiento de tallo (VT)}

El volcamiento de tallo (VT) presentó diferencias altamente significativas $(\mathrm{p}<0,01)$ para los cinco genotipos en todas las localidades de estudio. Los genotipos AV25-T y AV25-S presentaron los menores porcentajes de VT, sin diferir estadísticamente $(\mathrm{p}<0,01)$ entre sí en cada localidad, pero si fueron diferentes $(\mathrm{p}<0,01)$ a los testigos comerciales en todas las localidades. AV25-T y AV25-S presentaron valores de VT de 0,0 \% a 13,0 \% y 0,0\% a $17,5 \%$, respectivamente, a diferencia de los testigos comerciales que presentaron valores superiores, especialmente con la variedad Cayuse con 78,8 \% y 91,3\% en las localidades del Altiplano Cundiboyacense. Los genotipos comerciales Avenar y Cajicá presentaron valores de incidencia de volcamiento entre $20 \%$ y $60 \%$ (Cuadro 6).

\section{Roya de la hoja (RH) y del tallo (RT)}

En relación con roya de la hoja $(\mathrm{RH})$, los genotipos AV25-T y AV25-S presentaron valores de incidencia que no superaron el $12 \%$ en las dos subregiones, con una reacción resistente para la subregión altiplano Cundiboyacense y de moderadamente resistente a resistente para la subregión altiplano de Nariño. Las variedades Avenar y Cajicá presentaron valores de incidencia de $10 \%$ a $82 \%$ con una reacción moderadamente susceptible a susceptible en el altiplano Cundiboyacense y de $15 \%$ a $35 \%$ en la subregión altiplano de Nariño con una reacción moderadamente susceptible. Cayuse presentó los mayores valores de incidencia en el altiplano Cundiboyacense (60\% a 85\%) y en 
Cuadro 5. Días a cosecha de forraje verde (DCFV) de dos genotipos promisorios y tres variedades de avena forrajera (Avena sativa) en ocho localidades de la Zona Andina de Colombia. Corporación Colombiana de Investigación Agropecuaria (AGROSAVIA). 20162017.

Table 5. Days to harvest green forage (DCFV) of two promising genotypes and three varieties of forage oats (Avena sativa), in eight localities in the Colombian Andean zone. Corporación Colombiana de Investigación Agropecuaria (AGROSAVIA). $2016-2017$.

\begin{tabular}{lcccccccc}
\hline & \multicolumn{9}{c}{ Subregión/localidad } & \multicolumn{3}{c}{ Altiplano de Nariño } \\
\cline { 2 - 9 } Genotipo & MOS & DUI & CER & CUC & PAS & TAN & CUM & GUA \\
\cline { 2 - 9 } AV25-T & $119,3 \mathrm{~b}$ & $119,0 \mathrm{~b}$ & $124,0 \mathrm{~b}$ & $129,3 \mathrm{~b}$ & $144,0 \mathrm{~b}$ & $157,8 \mathrm{~b}$ & $111,0 \mathrm{~b}$ & $165,0 \mathrm{~b}$ \\
AV25-S & $119,3 \mathrm{~b}$ & $119,0 \mathrm{~b}$ & $125,0 \mathrm{~b}$ & $129,5 \mathrm{~b}$ & $144,0 \mathrm{~b}$ & $158,5 \mathrm{~b}$ & $111,0 \mathrm{~b}$ & $165,0 \mathrm{~b}$ \\
Avenar & $94,0 \mathrm{c}$ & $88,0 \mathrm{c}$ & $91,0 \mathrm{c}$ & $90,0 \mathrm{c}$ & $121,8 \mathrm{c}$ & $115,3 \mathrm{c}$ & $103,3 \mathrm{c}$ & $102,0 \mathrm{c}$ \\
Cajicá & $84,8 \mathrm{c}$ & $84,8 \mathrm{c}$ & $90,8 \mathrm{c}$ & $94,3 \mathrm{c}$ & $121,0 \mathrm{c}$ & $115,0 \mathrm{c}$ & $102,5 \mathrm{c}$ & $102,0 \mathrm{c}$ \\
Cayuse & $159,3 \mathrm{a}$ & $153,3 \mathrm{a}$ & $168,3 \mathrm{a}$ & $169,5 \mathrm{a}$ & $180,3 \mathrm{a}$ & $171,5 \mathrm{a}$ & $137,8 \mathrm{a}$ & $175,3 \mathrm{a}$ \\
\hline
\end{tabular}

a-b-c: Letras diferentes entre columnas indican diferencia estadística significativa $(\mathrm{p}<0,01)$. MOS: Mosquera; DUI: Duitama; CER: Cerinza; CUC: Cucunubá; PAS: Pasto; TAN: Tangua; CUM: Cumbal; GUA: Guachucal / a-b-c: Different letters between columns indicate significant statistical difference $(\mathrm{p}<0.01)$. MOS: Mosquera; DUI: Duitama; CER: Cerinza; CUC: Cucunubá; PAS: Pasture; TAN: Tangua; CUM: Cumbal; GUA: Guachucal.

Cuadro 6. Volcamiento de tallo $(\%)$ de dos genotipos promisorios y tres variedades de avena forrajera (Avena sativa) en ocho localidades de la Zona Andina de Colombia. Corporación Colombiana de Investigación Agropecuaria (AGROSAVIA), $2016-2017$.

Table 6. Stem overturning (\%) of two promising genotypes and three varieties of forage oats (Avena sativa) in eight localities in the Colombian Andean zone. Corporación Colombiana de Investigación Agropecuaria (AGROSAVIA), 2016-2017.

\begin{tabular}{|c|c|c|c|c|c|c|c|c|}
\hline \multirow[b]{3}{*}{ Genotipo } & \multicolumn{8}{|c|}{ Subregión/localidad } \\
\hline & \multicolumn{4}{|c|}{ Altiplano Cundiboyacense } & \multicolumn{4}{|c|}{ Altiplano de Nariño } \\
\hline & MOS & DUI & CER & CUC & PAS & TAN & CUM & GUA \\
\hline AV25-T & $8,8 \mathrm{c}$ & $10,0 \mathrm{c}$ & $10,0 \mathrm{c}$ & $0,0 \mathrm{~d}$ & $0,0 \mathrm{c}$ & $0,0 \mathrm{c}$ & $13,0 \mathrm{~b}$ & $0,0 \mathrm{~b}$ \\
\hline AV25-S & $17,5 \mathrm{c}$ & $9,3 \mathrm{c}$ & $8,8 \mathrm{c}$ & $1,8 \mathrm{~d}$ & $0,0 \mathrm{c}$ & $0,0 \mathrm{c}$ & $12,3 \mathrm{~b}$ & $0,0 \mathrm{~b}$ \\
\hline Avenar & $43,8 \mathrm{~b}$ & $33,8 \mathrm{~b}$ & $40,0 \mathrm{~b}$ & $11,3 \mathrm{c}$ & $20,8 \mathrm{~b}$ & $21,8 \mathrm{~b}$ & 65,0 a & $37,5 \mathrm{a}$ \\
\hline Cajicá & $60,0 \mathrm{~b}$ & $38,8 \mathrm{~b}$ & $50,0 \mathrm{~b}$ & $20,0 \mathrm{~b}$ & $20,0 \mathrm{~b}$ & $24,3 \mathrm{~b}$ & $62,5 \mathrm{a}$ & $38,8 \mathrm{a}$ \\
\hline Cayuse & 83,8 a & $78,8 \mathrm{a}$ & $91,3 \mathrm{a}$ & 78,8 a & $60,0 \mathrm{a}$ & $53,8 \mathrm{a}$ & $51,8 \mathrm{a}$ & $40,2 \mathrm{a}$ \\
\hline
\end{tabular}

a-b-c: Letras diferentes entre columnas indican diferencia estadística significativa $(p<0,01)$. MOS: Mosquera; DUI: Duitama; CER: Cerinza; CUC: Cucunubá; PAS: Pasto; TAN: Tangua; CUM: Cumbal; GUA: Guachucal / a-b-c: Different letters between columns indicate significant statistical difference ( $\mathrm{p}<0.01)$. MOS: Mosquera; DUI: Duitama; CER: Cerinza; CUC: Cucunubá; PAS: Pasture; TAN: Tangua; CUM: Cumbal; GUA: Guachucal.

el altiplano de Nariño (35\% a $65 \%$ ), con una reacción susceptible a altamente susceptible en la primera subregión y de moderadamente susceptible a susceptible en la segunda subregión (Cuadro 7).

En relación con la roya del tallo (RT), en el altiplano Cundiboyacense y en el altiplano de Nariño los genotipos AV25-T y AV25-S presentaron valores de incidencia que no superaron el $12 \%$, con una reacción desde tipo inmune hasta moderadamente resistente. Avenar y Cajicá presentaron valores desde $22 \%$ a $60 \%$ en el altiplano 
Cuadro 7. Incidencia (\%) y reacción a roya de la hoja (Puccinia coronata f. sp avenae) de dos genotipos promisorios y tres variedades de avena forrajera (Avena sativa) en ocho localidades de la zona andina colombiana. Corporación Colombiana de Investigación Agropecuaria (AGROSAVIA). 2016-2017.

Table 7. Incidence (\%) and leaf rust reaction (Puccinia coronata f. sp avenae) of two promising genotypes and three varieties of forage oats (Avena sativa) in eight localities in the Colombian Andean zone. Corporación Colombiana de Investigación Agropecuaria (AGROSAVIA). 2016-2017.

\begin{tabular}{llccccc}
\hline Subregión & Localidad & AV25-T & AV25-S & Avenar & Cajicá & Cayuse \\
\hline Altiplano & Mosquera & $2-5 *$ & $1-12$ & $20-60$ & $20-70$ & $60-85$ \\
Cundiboyacense & Duitama & $1-2$ & $1-2$ & $20-60$ & $60-82$ & $60-85$ \\
& Cerinza & $1-5$ & $1-5$ & $20-60$ & $20-60$ & $60-82$ \\
& Cucunubá & $2-5$ & $2-5$ & $10-40$ & $20-60$ & $60-85$ \\
\hline Tipo de reacción & & R & R & MS-S & MS-S & S-AS \\
\hline Altiplano de Nariño & Pasto & $8-11$ & $8-10$ & $20-25$ & $30-35$ & $30-60$ \\
& Tangua & $8-11$ & $9-11$ & $15-25$ & $25-35$ & $40-50$ \\
& Cumbal & $10-11$ & $11-12$ & $20-25$ & $30-35$ & $40-65$ \\
& Guachucal & $11-12$ & $9-10$ & $20-30$ & $25-35$ & $35-45$ \\
\hline Tipo de reacción & & R-MR & R-MR & MS & MS & MS-S \\
\hline
\end{tabular}

(*): menor y mayor valor de incidencia en porcentaje; R: resistente; MR: moderadamente resistente; MS: moderadamente susceptible; S: susceptible / (*): lower and higher incidence value in percentage, R: resistant; MR: moderately resistant; MS: moderately susceptible; S: susceptible.

Cundiboyacense y de $10 \%$ a $22 \%$ en el altiplano de Nariño, con una reacción moderadamente susceptible a susceptible en la primera subregión y de moderadamente resistente en la segunda subregión. La variedad Cayuse presentó los mayores valores de incidencia en ambas subregiones (25\% a $70 \%$ en el altiplano Cundiboyacense y de $15 \%$ a $30 \%$ en el altiplano de Nariño), con una reacción susceptible para la primera subregión y de moderadamente resistente a moderadamente susceptible en la segunda subregión (Cuadro 8).

\section{Rendimiento de forraje verde (RFV)}

De acuerdo con el análisis de varianza combinado a través de localidades para rendimiento de forraje verde (RFV), se presentaron diferencias significativas para localidad, genotipo e interacción genotipo x localidad. La mayor participación de la variación total del RFV se debió a los componentes de localidad y genotipo, con un valor de $51,0 \%$ y 43,7 \%, respectivamente, y la menor participación a la interacción genotipo x localidad $(8,0 \%)$. Con base en el análisis AMMI, se estableció mediante la prueba de Gollob (1968), que los dos primeros componentes principales, con una significancia de $\alpha<0,01$, explicaron el 78,7 \% de la interacción genotipo x localidad (Cuadro 9). Este resultado permite una interpretación confiable del comportamiento de la interacción genotipo x localidad, ya que un valor aceptable es una proporción mayor a $75 \%$ (Crossa et al., 2002). El coeficiente de variación observado fue de $11,7 \%$, el cual es considerado aceptable. La mayor participación del factor localidad en la variación total del modelo estadístico para RFV se debió posiblemente al déficit de agua observado en dos de las ocho localidades del estudio (TAN y DUI) que no lograron satisfacer el $100 \%$ del requerimiento de agua.

El gráfico biplot del modelo AMMI obtenido con base en los dos primeros componentes principales: CP1 y CP2 (Figura 1), permitió el reconocimiento de genotipos con adaptación específica a aquellos asociados al vértice del vector de una o varias localidades y el aporte de cada localidad en la interacción genotipo x localidad (mayor 
Cuadro 8. Incidencia (\%) y reacción a roya del tallo (Puccinia graminis f. sp avenae Erikss y Henning) de dos genotipos promisorios y tres testigos comerciales de avena forrajera (Avena sativa) en ocho localidades de la zona andina colombiana. Corporación Colombiana de Investigación Agropecuaria (AGROSAVIA). 2016-2017.

Table 8. Incidence (\%) and stem rust (Puccinia graminis f. sp avenae Erikss and Henning) reaction of two promising genotypes and three commercial control of forage oats (Avena sativa) in eight localities in the Colombian Andean zone. Corporación Colombiana de Investigación Agropecuaria (AGROSAVIA). 2016-2017.

\begin{tabular}{llccccc}
\hline Subregión & Localidad & AV25-T & AV25-S & Avenar & Cajicá & Cayuse \\
\hline Altiplano & Mosquera & $0-2$ & $5-10$ & $22-40$ & $25-40$ & $40-70$ \\
Cundiboyacense & Duitama & $0-2$ & $10-12$ & $25-30$ & $25-60$ & $30-70$ \\
& Cerinza & $0-2$ & $10-12$ & $22-40$ & $25-60$ & $30-70$ \\
& Cucunubá & $0-2$ & $5-10$ & $25-60$ & $25-30$ & $25-50$ \\
\hline Tipo de reacción & & I-MR & R-MR & MS-S & MS-S & S \\
Altiplano de Nariño & Pasto & $0-5$ & $0-5$ & $15-20$ & $15-20$ & $15-20$ \\
& Tangua & $0-5$ & $0-5$ & $10-20$ & $15-20$ & $20-25$ \\
& Cumbal & $0-10$ & $5-8$ & $10-15$ & $15-20$ & $25-30$ \\
& Guachucal & $0-5$ & $0-5$ & $15-20$ & $15-22$ & $15-20$ \\
\hline Tipo de reacción & & I-R & I-R & MR & MR & MR-MS \\
\hline
\end{tabular}

(*): menor y mayor valor de incidencia en porcentaje; I: inmune; R: resistente; MR: moderadamente resistente; MS: moderadamente susceptible; S: susceptible / (*): lower and higher incidence value in percentage; I: immune; R: resistant; MR: moderately resistant; MS: moderately susceptible; S: susceptible.

Cuadro 9. Análisis AMMI para el rendimiento de forraje verde (RFV) de dos genotipos promisorios y tres testigos comerciales de avena forrajera (Avena sativa) en ocho localidades de la zona andina colombiana. Corporación Colombiana de Investigación Agropecuaria (AGROSAVIA). 2016-2017.

Table 9. AMMI analysis for the green forage yield (RFV) of two promising genotypes and three commercial control forage oats (Avena sativa) in eight localities in the Colombian Andean area. Corporación Colombiana de Investigación Agropecuaria (AGROSAVIA). 2016-2017.

\begin{tabular}{lccc}
\hline Fuente de variación & g.l & Cuadrado medio (C.M.) & \% \\
\hline Localidad - L & 7 & $7917,8 * *$ & $51,0(1)$ \\
Repetición (L) & 3 & 91,6 & $43,7(1)$ \\
Genotipo - G & 4 & $2661,2^{* *}$ & $8,0(1)$ \\
G x L & 28 & $222,2 * *$ & $54,1(2)$ \\
CP1 & 10 & $3366,8^{* *}$ & $78,7(2)$ \\
CP2 & 8 & $1533,2 * *$ & $96,7(2)$ \\
CP3 & 4 & $1119,5 \mathrm{~ns}$ & $100,0(2)$ \\
CP4 & 2 & $203,1 \mathrm{~ns}$ & 46,0 \\
Error & 117 & 11,7 & \\
C.V $(\%)$ & & & \\
\hline
\end{tabular}

** Diferencias significativas $(\alpha<0,01)$ para localidad y genotipo; **, ns Diferencias significativas $(\alpha<0,01)$ y no significativas (Prueba de Gollob) para componentes principales CP1 y CP2. (1): porcentaje de participación en la variación total; (2): porcentaje acumulado / ** Significant differences $(\alpha<0.01)$ for locality and genotype; **, ns Significant differences $(\alpha<0.01)$ and non-significant differences (Gollob test) for main components CP1 and CP2. (1): percentage of participation in the total variation; (2): accumulated percentage. 


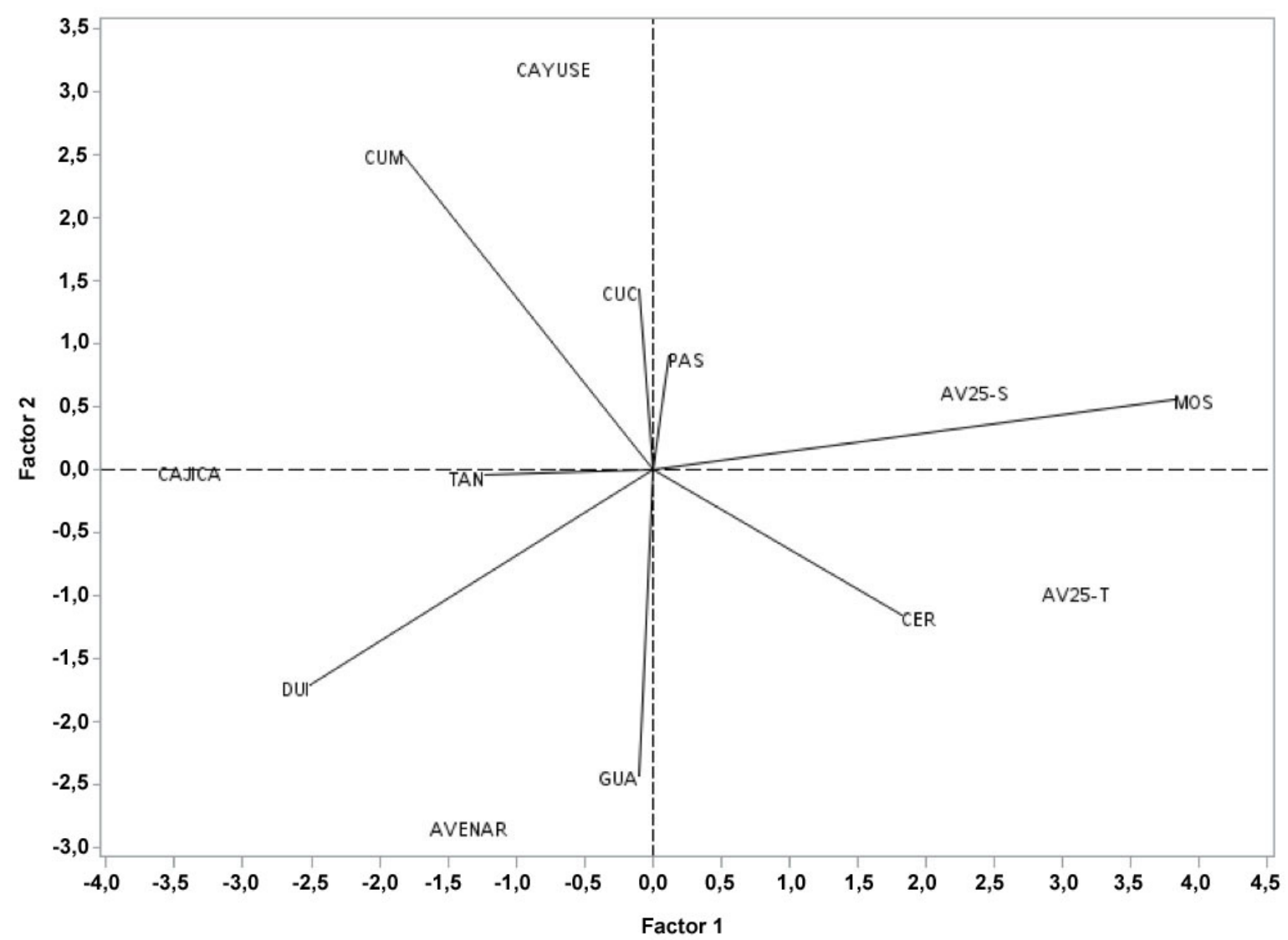

Figura 1. Representación de genotipos y ambientes respecto a los dos primeros componentes CP1 y CP2, del análisis AMMI para el rendimiento de forraje verde de dos genotipos y tres testigos comerciales de avena forrajera (Avena sativa) evaluados en ocho ambientes de la zona andina colombiana. Corporación Colombiana de Investigación Agropecuaria (AGROSAVIA). 2016-2017.

MOS: Mosquera; CUC: Cucunubá; DUI: Duitama; CER: Cerinza; PAS: Pasto; TAN: Tangua; CUM: Cumbal; GUA: Guachucal.

Figure 1. Representation of genotypes and environments with respect to the first two components CP1 and CP2 of the AMMI analysis for the green forage yield of two genotypes and three commercial controls of forage oats (Avena sativa) evaluated in eight environments of the Colombian Andean area. Corporación Colombiana de Investigación Agropecuaria (AGROSAVIA). 2016-2017.

MOS: Mosquera; CUC: Cucunubá; DUI: Duitama; CER: Cerinza; PAS: Pasto; TAN: Tangua; CUM: Cumbal; GUA: Guachucal.

longitud del vector mayor aporte en la interacción y viceversa), así como la potencia para discriminar genotipos. Por su mayor y menor longitud del vector localidad, los ambientes MOS, DUI, CUM fueron los de mayor aporte a la interacción genotipo x localidad y PAS, CUC y TAN los de menor aporte.

La adaptación específica se observó en AV25-T y AV25-S asociado con las localidades PAS, MOS y CER; Avenar con DUI y GUA; Cajicá con CUM, DUI y TAN y Cayuse con CUM. En relación con la potencia para estratificación del RFV en $\mathrm{t} \mathrm{ha}^{-1}$ por su mayor longitud del vector fueron MOS, CUM y DUI, con valores desde 47,8 a 92,5, y la menor potencia de estratificación PAS con valores de 36,5 a 63,5 (Figura 1). Las localidades MOS y CER presentaron RFV de 83,0 para CER y 74,0 para MOS, sin diferir estadísticamente entre sí y diferente estadísticamente con las otras localidades (Cuadro 10). Por su alto rendimiento y mayor longitud del vector estas 
Cuadro 10. Rendimiento de forraje verde (RFV) $\left(\mathrm{t} \mathrm{ha}^{-1}\right)$ de dos genotipos promisorios y tres variedades de avena forrajera (Avena sativa), en ocho localidades de la zona andina colombiana. Corporación Colombiana de Investigación Agropecuaria (AGROSAVIA). 2016-2017.

Table 10. Green forage yield (RFV) ( $\left(\mathrm{ha}^{-1}\right)$ of two promising genotypes and three varieties of forage oats (Avena sativa) in eight localities in the Colombian Andean area. Corporación Colombiana de Investigación Agropecuaria (AGROSAVIA). $2016-2017$.

\begin{tabular}{|c|c|c|c|c|c|c|c|c|}
\hline \multirow[b]{3}{*}{ Genotipo } & \multicolumn{8}{|c|}{ Subregión/localidad } \\
\hline & \multicolumn{4}{|c|}{ Altiplano Cundiboyacense } & \multicolumn{4}{|c|}{ Altiplano de Nariño } \\
\hline & MOS & DUI & CER & CUC & PAS & TAN & CUM & GUA \\
\hline AV25-T & $92,5 \mathrm{a}$ & $30,0 \mathrm{a}$ & $93,1 \mathrm{a}$ & $57,8 \mathrm{a}$ & $62,5 \mathrm{a}$ & $34,5 \mathrm{a}$ & $64,7 \mathrm{ab}$ & $81,8 \mathrm{a}$ \\
\hline AV25-S & $92,5 \mathrm{a}$ & $28,7 \mathrm{a}$ & 99,3 a & 56,0 a & $56,6 \mathrm{~b}$ & $31,0 \mathrm{a}$ & 79,9 a & $78,2 \mathrm{~b}$ \\
\hline Avenar & $64,3 \mathrm{~b}$ & $37,5 \mathrm{a}$ & $82,7 \mathrm{ab}$ & $45,5 \mathrm{~b}$ & $52,1 \mathrm{ab}$ & $27,5 \mathrm{ab}$ & $60,5 \mathrm{~b}$ & $73,9 \mathrm{~b}$ \\
\hline Cajicá & $47,8 \mathrm{c}$ & $20,5 \mathrm{~b}$ & $61,8 \mathrm{c}$ & $34,7 \mathrm{c}$ & $36,5 \mathrm{c}$ & $25,9 \mathrm{c}$ & $62,7 \mathrm{ab}$ & $59,8 \mathrm{c}$ \\
\hline Cayuse & $73,5 \mathrm{~b}$ & $29,3 \mathrm{a}$ & $78,3 \mathrm{~b}$ & $58,8 \mathrm{a}$ & $63,5 \mathrm{a}$ & $29,6 \mathrm{ab}$ & $76,3 \mathrm{ab}$ & $63,5 \mathrm{ab}$ \\
\hline $\begin{array}{l}\text { Promedio } \\
\text { localidad }\end{array}$ & $74,1 \mathrm{a}$ & $29,2 \mathrm{~d}$ & 83,0 a & $44,6 \mathrm{c}$ & $54,2 \mathrm{c}$ & $29,7 \mathrm{~d}$ & $68,8 \mathrm{~b}$ & $71,4 \mathrm{a}$ \\
\hline
\end{tabular}

a-b-c: Different letters between columns and row for average locality indicate significant statistical difference $(\mathrm{p}<0.01))$. MOS: Mosquera; DUI: Duitama; CER: Cerinza; CUC: Cucunubá; PAS: Pasto; TAN: Tangua; CUM: Cumbal; GUA: Guachucal / a-b-c: Different letters between columns indicate significant statistical difference ( $\mathrm{p}<0.01)$. MOS: Mosquera; DUI: Duitama; CER: Cerinza; CUC: Cucunubá; PAS: Pasture; TAN: Tangua; CUM: Cumbal; GUA: Guachucal.

dos localidades se constituyeron en los mejores ambientes de selección para el programa de mejoramiento genético de avena forraje para AGROSAVIA en Colombia.

En todos los casos, el RFV de AV25-T y AV25-S fue superior a las variedades testigo con los mayores rendimientos en CER (93,1 y 99,3) y los menores en DUI (30,0 y 28,7). AV25-T superó en 9,4\%, 21,2 \% y 5,8 $\%$ a Avenar, Cajicá y Cayuse, respectivamente y AV25-S supero en $11,0 \%, 22,8 \%$ y 7,4 \% a Avenar, Cajicá y Cayuse, respectivamente.

\section{Discusión}

Las localidades del estudio ubicadas en dos de las tres principales cuencas lecheras de Colombia: Altiplano Cundiboyacense y Altiplano de Nariño (Garcés et al., 2000), presentaron suelos caracterizados por su origen de tipo volcánico como Inceptisol (Ramos, 2017), con un nivel de fertilidad de media a alta por sus contenidos de materia orgánica y de fósforo, que permitieron obtener, en seis de las ocho localidades, un rendimiento de forraje verde (RFV) de 57,8 a 93,1 t ha-1. Es reconocido a nivel mundial que el mejoramiento genético en avena es una de las estrategias efectivas para lograr incrementos significativos en el rendimiento de forraje, grano y resistencia a las royas y tolerancia al volcamiento. El rendimiento de forraje verde (RFV), de AV25-T y AV25-S fue superior al promedio nacional reportado en Colombia de $40 \mathrm{t} \mathrm{ha}^{-1}$ (Terán-Chaves et al., 2014) y al reportado en India de 35 a 50 (Singh et al., 2018), en Etiopía de 40 a 67 (Beyene et al., 2015) y en Pakistán de 42 a 75 (Lodhi et al., 2009; Numan et al., 2016), e igualmente superior a los valores asociados con el óptimo productivo reportado para esta especie de 60 a 80 (Haqqani et al., 2003). Asimismo, se consideraron similares al reportado por Naeem et al. (2002) en la India de 80 a 84 y menor a las $116 \mathrm{t} \mathrm{ha}^{-1}$ reportado en zonas altas de Etiopia (Semman et al., 2018).

Los genotipos AV25-T y AV25-S, se constituyen en una oferta genética que permitirá en los próximos años incrementar el promedio de RFV a nivel país; sin embargo, se reconoce que, en Colombia, aún queda una brecha en 
el RFV para cerrar por la vía del mejoramiento genético. Lo anterior está relacionado, con la significativa ganancia genética del $35 \%$ a través del mejoramiento a nivel mundial durante 33 años (1983 a 2012) (Laidig et al., 2014). El AV25-T y AV25-S son producto del mejoramiento genético del Centro Internacional de Mejoramiento de Maíz y Trigo (CIMMYT), y su introducción y selección en condiciones del Trópico Alto colombiano podrían mejorar el promedio nacional de $50 \mathrm{t} \mathrm{ha}^{-1}$.

El comportamiento de los genotipos AV25-T y AV25-S, aún en condiciones de déficit de agua, mostró un RFV en $t$ ha ${ }^{-1}$ de 57,8 y 34,5 en CUC y TAN, respectivamente. El efecto del déficit del agua en avena esta reportada con datos obtenidos durante 33 años (1980-2012), donde la precipitación fue la responsable entre el $12 \%$ y el $50 \%$ de la variación del rendimiento de avena en Minessota (EE. UU) (Klink et al., 2013); efecto que podría ser explicado en este estudio en la mayor participación de la variación total del rendimiento de forraje verde en el modelo estadístico del factor localidad del 51,0\%. No obstante, la interacción genotipo x ambiente al ser significativa, permitió un desempeño diferencial de los genotipos a través de las localidades para esta variable y reconocer mediante el gráfico biplot el grado de asociación con localidades o adaptación específica.

El grado de incidencia bajo y reacción tipo resistente en AV25-T y AV25-S permitirá la repetibilidad del desempeño en condicionales comerciales en cada subregión específica de recomendación.

En el caso específico de Colombia, la roya de la hoja y del tallo son igualmente los patógenos más dañinos, afectan la avena desde estados de desarrollo tempranos con reducciones del $75 \%$ en el RFV y de $60 \%$ en grano (Leyva-Mir et al., 2004; Berlín et al., 2013), y pérdidas del 5 al 34 \% en la producción de materia seca en variedades susceptibles (Leyva-Mir et al., 2018). Los estudios reportan que la genética de la resistencia a la roya de la hoja y del tallo en avena es de herencia simple y está condicionada por uno a dos genes con un tipo de acción dominante. No obstante, de no conocerse con detalle los genes asociados a la resistencia de los genotipos AV25-T y AV25-S por el tipo de reacción, se deduce que es de tipo vertical (gen-gen).

Teniendo en cuenta los tres atributos de desempeño de un genotipo para que sea candidato a variedad: alto RFV, resistencia a roya de la hoja y del tallo y tolerancia al volcamiento, AV25-T y AV25-S los cumplieron satisfactoriamente, además de demostrar un nivel de precocidad en DF y DCFV significativamente menor a la variedad más tardía Cayuse. No obstante, fue seleccionada AV25-T por presentar menor incidencia de roya del tallo en la subregión Altiplano Cundiboyacense e inscrita en el sistema nacional de cultivares como nueva variedad forrajera denominada Altoandina para la subregión Altiplano Cundiboyacense y Altiplano de Nariño.

\section{Conclusiones}

Se inscribió el genotipo AV25-T en el sistema nacional de cultivares como nueva variedad forrajera denominada Altoandina, con base en la prueba de evaluación de rendimiento realizada en ocho localidades de la región Andina, subregión natural del Altiplano Cundiboyacense y Altiplano de Nariño, durante el período 2016-2017, se seleccionó por su tolerancia a volcamiento, resistencia a la roya de la hoja y del tallo y rendimiento de forraje verde superior a las variedades comerciales.

\section{Literatura citada}

Arango, J., F.A. Cardona, A. López, G. Correa, y J.J. Echeverry. 2017. Variación de caracteres morfológicos del pasto kikuyo (Cenchrus clandestinus) en el trópico alto de Antioquia. CES Med. Vet. Zootec. 12(1):44-52. doi:10.21615/cesmvz.12.1.4

Berlín, A., B. Samils, A. Djurle, H. Wirsén, L. Szabo, and J. Yuen. 2013. Disease development and genotypic diversity of Puccinia graminis f. sp. Avenae in Swedish oat fields. Plant Pathol. 62(1):32-40. doi:10.1111/j.1365-3059.2012. 02609.x 
Beyene, G., A. Araya, and H. Gebremedhin. 2015. Evaluation of different oat varieties for fodder yield and yield related traits in Debre Berhan Area, Central Highlands of Ethiopia. Livest. Res. Rural Dev. 27:170. http://www.lrrd.org/lrrd27/9/ gebr27170.htm (consultado abril 2019).

Bhatti, M.B.A., A. Hussain, and D. Muhammad. 1992. Potential for forage production of different varieties of oats under two cutting systems. Pak. J. Agric. Res. 13(2):184-190.

Carulla, J.E., y E. Ortega. 2016. Sistemas de producción lechera en Colombia: retos y oportunidades. Arch. Latinoam. Prod. Anim. 24(2):83-87.

Crossa, J., P. Cornelius, and W. Yan. 2002. Biplots of linear-bilinear models for studying crossover genotype environment interaction. Crop. Sci. 42:619-633. doi:10.2135/cropsci2002.0619

Cuesta, P.A. 2005. Fundamentos de manejo de praderas para mejorar la productividad de la ganadería del trópico colombiano. Cinenc. Tecnol. Agropecu. 6(2):5-18. doi:10.21930/rcta.vol6_num2_art:42

DANE (Departamento de Administracion Nacional de Estadística). 2015. Encuesta nacional agropecuaria. DANE. https://www. dane.gov.co/files/investigaciones/agropecuario/enda/ena/2015/presentacion_ena_2015.pdf (consultado feb. 2019).

Gabriel, K. 1971. The biplot graphic of matrices with application to principal component analysis. Biometrics 58:453-467. doi: $10.2307 / 2334381$

Garcés, A.M., L. Berrio, S. Ruiz, J.G. Serna, y A.F. Builes. 2000. Ensilaje como fuente de alimentación para el ganado. Rev. Lasallista Investig. 1(1):66-71.

Gollob, H.F. 1968. A statistical model which combines features of factor analytic and analysis of variance techniques. Psychometrika 33:73-115. doi:10.1007/BF02289676

Gutiérrez, H.J. 1991. Clasificaciones climáticas. HIMAT, Bogotá, Colombia.

Haqqani, A, M., Z. Ali, S. Shafiqe, S. Zahid, and A. Baksh. 2003. Oats: A fodder of winter lean period. Agric-Digest. Zari Traqiati Bank Limited. 23:15-23.

Hussain, A., S. Khan, A. Bakhsh, M. Imran, and M. Ansar. 2010. Variability in fodder production potential of exotic oats (Avena sativa) genotypes under irrigated conditions. J. Agric. Res. 48:65-71.

ICA (Instituto Colombiano Agropecuario). 2000. Resolución 1985: Pruebas de evaluación agronómica. ICA. https://www.ica. gov.co/getattachment/1e3dbbcd-e900-4c3d-9474-0c98dfbf5f48/2000R1985 aspx (consultado 5 abril. 2019).

IDEAM (Instituto de Hidrología, Meteorología y Estudios Ambientales). 2017. Clasificaciones climáticas Colombia. IDEAM. http://www.ideam.gov.co/documents/21021/21789/climas+\%5BModo+de+compatibilidad\%5D.pdf/d8c85704-a07a4290-ba65-f2042ce99ff9 (consultado abril 2019).

Keiper, F.J., M.S. Haque, M.J. Hayden, and R.F. Park. 2006. Genetic diversity in Australian populations of Puccinia graminis f. sp. avenae. Phytopathology 96(1):96-104. doi:10.1094/PHYTO-96-0096

Klink, K., J.J. Wiersma, C.J. Crawford, and D.D. Stuthman. 2013. Impacts of temperature and precipitation variability in the Northern plains of the United States and Canada on the productivity of spring barley and oat. Int. J. Climatol. 34:28052818. doi:10.1002/joc.3877

Laidig, F., H. P. Piepho, T. Drobek, and U. Meyer. 2014. Genetic and non-genetic trends of 12 different crops in yield tests of official German varieties and yield trends on the farm. Theor. Appl. Genet. 127:2599-2617. doi:10.1007/s00122-014-2402-z

Leyva-Mir, S.G., E. Espitia-Rangel, H.E. Villaseñor-Mir, y J. Huerta-Espino. 2004. Pérdidas ocasionadas por Puccinia graminis f. sp. avenae Ericks. y Henn., causante de la roya del tallo en seis cultivares de avena (Avena sativa L.) en los Valles Altos de México. Rev. Mex. Fitopatol. 22:166-171. 
Leyva-Mir, S.G., H.E. Villaseñor-Mir, M. Camacho-Tapia, G. D. Ávila-Quezada, E. García-León, y J. M. Tovar-Pedraza. 2018. Respuesta de genotipos de avena a la infección por Puccinia graminis f. sp. avenae en Valles Altos de México. Rev. Mex. Cienc. Agríc. 9:317-328. doi:10.29312/remexca.v9i2.1074

Lodhi, M.Y., I.B. Marghazani, K. Hamayun and M.J. Marri. 2009. Comparative performance study of different oat varieties under agro-climatic conditions of Sibi. J. Anim. Plant. Sci. 19(1):34-36.

Mamani, J., y F.H. Cotacallapa. 2018. Rendimiento y calidad nutricional de avena forrajera en la región de Puno. Rev. Investig. Altoandin. 20:384-400. doi:10.18271/ria.2018.415

Montaño-Carrasco, M., A. Hernández-Rodríguez, A. Núñez-Barros, y V. Guerrero-Prieto. 2017. Producción y contenido nutrimental en avena forrajera fertilizada con fuentes químicas y orgánicas. Rev. Fitotec. Mex. 40:317-324.

Naeem, M. A.K. Muhammad, M. Shahid Munir Chohan, A. Hassan Khan, and S. Salahuddin. 2002. Evaluation of different varieties of oats for green fodder yield potential. Asian J. Plant Sci. 1:640-641. doi:10.3923/ajps.2002.640.641

Numan, Ali., Z. A. Muhammad, J. Tariq, F. M. Shamsher and A. Saleem. 2016. Assessing yield and yield associated traits of oat genotypes grown under the semi-arid conditions of Pakistán. American-Eurasian J. Agric. Environ. Sci. 16:1784-1789. doi:10.5829/idosi.aejaes.2016.1784.1789

Peterson, R.F., A.B. Campbell, and A.E. Hannah. 1948. A diagrammatic scale for estimating rust intensity on leaves and stems of cereals. Can. J. Res. 26:496-500. doi:10.1139/cjr48c-033

Ramos, A. 2017. Identificación de suelos del orden Inceptisol. Rev. Logos Cienc. Tecnol. 8(2):170-181. doi:10.22335/rlct. v8i2.304

Ramírez-Ordóñez, S., D. Domínguez-Díaz, J. Salmerón-Zamora, G. Villalobos-Villalobos, y J. Ortega-Gutiérrez. 2013. Producción y calidad del forraje de variedades de avena en función del sistema de siembra y de la etapa de madurez al corte. Rev. Fitotec. Mex. 36:395-403.

SAS Institute Inc. 2003. User's guide Version 9.3. SAS Institute Inc., Cary, NC, USA.

Semman, S., B. Eba, and T. Dinkale. 2018. Performance evaluation of improved oat varieties/accessions at the highland of Guji Zone, Bore, Ethiopia. J. Biol. Agric. Healthcare 8(17):21-26.

Singh, D., A. Chauhan, and A. Chaudhary. 2018. Relative performance of oat forage varieties for seed production, economics and fodder yield under central Gujarat conditions. Forage Res. 44(3):185-191. doi:10.13140/RG.2.2.14349.38888

Sossa, C.P., y R. Barahona. 2015. Comportamiento productivo de novillos pastoreando en trópico de altura con y sin suplementación energética. Rev. Med. Vet. Zootec. 62:67-80. doi:10.15446/rfmvz.v62n1.49386

Terán-Chaves, C., G. Murcia, y A. García-Prats. 2014. Determinación de requerimientos hídricos para avena forrajera (Avena sativa L.) y raigrás (Lolium perenne) en la Sabana de Bogotá (Colombia). Rev. Fac. Nal. Agr. Medellín 67:1157-1158.

Vargas, J.J., A.M. Sierra., E.A. Mancipe., y Y. Avellaneda. 2018. El kikuyo, una gramínea presente en los sistemas de rumiantes en trópico alto colombiano. CES Med. Vet. Zootec. 13:137-156. doi:10.21615/cesmvz.13.2.4

Wise, R.P., and K.S. Gobleman-Werner. 1993. Resistance to oat crown rust in diploid and hexaploid Avena. Plant Dis. 77:355358. doi:10.1094/PD-77-0355

Zadocks, J.C., T.T. Chang, and C.F. Konza. 1974. A decimal code for the growth stages of cereals. Weed Res. 14:415-421. doi:10.1111/j.1365-3180.1974.tb01084.x 